\title{
The HKIE Best Transactions Paper Prize 2020
}

The HKIE Best Transactions Paper Prize 2020 is awarded to a distinguished paper nominated from the papers published in Volume 26 Number 2, Volume 26 Number 3 and Volume 27 Number 1 of HKIE Transactions. The prize is composed of a plaque, a certificate and a cash prize of $\operatorname{HK} \$ 5,000$ for the awarded paper.

This year, the HKIE Best Transactions Paper Prize is awarded to:

"Acidogenic phosphorus recovery from iron-facilitated primary sedimentation sludge in wastewater treatment" by Ruohong LI, Xiaoyan LI (published in HKIE Transactions Volume 26 Number 2)

Congratulations to our distinguished authors! The paper can be accessed on the HKIE website at http://www.hkie.org.hk/hkietransactionsonlineaccess.

Authors are welcome to submit your manuscripts to the HKIE Transactions ScholarOne Manuscripts site at $\mathrm{http}: / / \mathrm{mc} . \mathrm{manuscriptcentral.com/thie.}$

All papers published in HKIE Transactions will automatically enter the annual selection process of the HKIE Best Transactions Paper Prize. Up to three prizes will be given annually, and only if there are outstanding papers which warrant the awards. The Panel of Judges reserves the right to withhold any or all of the awards at their absolute discretion. 